\title{
Laryngectomy: what is the impact of the type of surgery on life quality and sexual function?
}

\author{
Laringectomia; qual è l'impatto del tipo di chirurgia sulla qualità di vita \\ e sulla sessualità? \\ F. AKIL ${ }^{1}$, U. YOLLU², S.F. TOPRAK ${ }^{3}$, M. AYRAL ${ }^{3}$ \\ ${ }^{1}$ Diyarbakir Selahaddin Eyyubi Public Hospital, Otolaryngology Clinic, Diyarbakir, Turkey; ${ }^{2}$ Gumushane Public \\ Hospital, Otolaryngology Clinic, Gumushane, Turkey; ${ }^{3}$ Diyarbakir Gazi Yasargil, Education and Research Hospital, \\ Otolaryngology Department, Diyarbakir, Turkey
}

\begin{abstract}
SUMMARY
The purpose of this study is to compare total and partial larygectomy on private life functions and sexuality in patients with laryngeal cancer. The study included 31 partial laryngectomy patients (Group 1) and 51 total laryngectomy patients (Group 2) who were operated for laryngeal cancer. European Organization for Research and Treatment of Cancer (EORTC) head and neck cancer module (QLQ-H\&N35) and Arizona Sexual Experiences Scale (ASEX) forms were filled in by interviewing face to face with patients. HNSW (swallowing), HNSE (senses), HNSP (speech), HNSO (social eating), HNSX (sexuality), HNTE (problems with teeth), HNOM (problems with opening mouth) and HNCO (coughing) scores of EORTC QLQ-H\&N35 were significantly higher in group 2 than in group 1. However, according to Arizona test results, there were no significant difference between the two groups.
\end{abstract}

KEY WORDS: Laryngectomy • Life quality • Sexuality • EORTC QLQ-H\&N35・Arizona Sexual Experiences Scale

\section{RIASSUNTO}

Lo scopo del presente studio è stato quello di confrontare gli effetti della laringectomia totale e della laringetomia parziale sulla qualità di vita e sulla sessualità. Lo studio ha incluso 31 pazienti affetti da carcinoma laringeo sottoposti a laringectomia parziale (Gruppo 1) e 51 pazienti sottoposti a laringectomia totale (Gruppo 2). Ai pazienti sono stati somministrati i questionari dedicati dell' EORTC (QLQ-H\&N35) e l'Arizona Sexual Experiences Scale (ASEX). I risultati del test EORTC QLQ-H\&N35 relativamente alle sezioni HNSW (deglutizione), HNSE (sensi), HNSP (voce), HNSO (alimentazione sociale), HNSX (sessualità), HNTE (problematiche coi denti), HNOM (problemi nell'apertura della bocca) e HNCO (tosse) sono risultati significativamente più alti per il gruppo 2. Tuttavia il test Arizona non ha mostrato differenze significative fra i due gruppi.

PAROLE CHIAVE: Laringectomia $\bullet$ Qualità di vita $\bullet$ Sessualità $\bullet$ EORTC QLQ-H\&N35・Arizona Sexual Experiences Scale

Acta Otorhinolaryngol Ital 2017;37:276-280

\section{Introduction}

Head and neck cancers, and particularly cancers of the larynx, is an issue that affects the daily lives of patients with severe consequences according to the kind of surgery that was performed. Choice of treatment depends on stage at diagnosis; organ sparing surgery such as partial laryngectomy is performed in early stages and total laryngectomy in advanced cases. In order to provide valuable information on how the type of surgery impacts the quality of life and sexual function, we divided our patients into two categories: patients that received partial laryngectomy in which the speech organ, smell sense, taste sense and air passage are preserved and patients who received total laryngectomy in which none of these were preserved. To compare the quality of life in these two patient groups and to investigate the psychophysiological effects objectively, we used European Organization for Research and Treatment of Cancer (EORTC) head and neck cancer module (QLQ-H\&N35) which was first established in $1999^{1}$, and we also used Arizona Sexual Experiences Scale to compare the sexual functions of both patient groups.

\section{Materials and methods}

\section{Study design}

This is a cross-sectional study. The protocols were approved by the Cerrahpasa Medical Faculty Ethics Committee at Istanbul University. All patients were promised privacy and all patients who were included consented to the study. 
Sample

The inclusion criteria for total laryngectomy patients included having completed treatment at least four months before the study, and for total laryngectomy patients, having been decannulated at least four months before the study. Patients with residual or recurrent tumour, psychiatric disease, diabetes or major heart disease, or who declined a face-to-face interview were excluded. The initial sample set consisted of 127 patients who underwent surgery for laryngeal cancer between 2010 and 2013 at the otolaryngology department of single tertiary cancer center. Female patients formed a relatively small number and were excluded in order to obtain a homogenous sample set. The remaining 82 male patients were divided by surgical procedure: partial laryngectomy (Group 1; 31 patients) and total laryngectomy (Group 2; 51 patients). The same clinician carried out all face-to-face interviews. All patients completed the head and neck cancer module of the quality of life questionnaire (QLQ-H\&N35) of the European Organization for Research and Treatment of Cancer (EORTC), as well as the Arizona Sexual Experiences Scale (ASEX). Additional demographic data were recorded, including educational level, monthly income, post-operative follow-up duration, treatment modality, and tumour, node and metastases (TNM) stage.

\section{Instruments}

ASEX is a questionnaire consisting of five sections on sexual function and a scoring scale between 1 and 5. The sections cover sexual drive, erection, arousal, orgasm and satisfaction. Patients are considered to have severe sexual dysfunction if they score 5 or 6 on a single section, 4 or higher in 3 or more sections, or 19 or more in total ${ }^{2}$. Both patient groups were evaluated and compared for average scores (ASEX1) and the ratios of patients with sexual dysfunction (ASEX2) were calculated.

The QLQ-H\&N35 was developed as an addition to the EORTC QLQ C-30, in order to better evaluate the quality of life of cancer patients ${ }^{13}$. It consists of 35 questions forming 18 measurements. A lower score indicates better quality of life ${ }^{4}$.

\section{Statistical analysis}

Statistical analysis was performed using IBM SPSS software version 16.0. Means and standard deviations were calculated for demographic data and questionnaire scores. Group comparisons were made with Student's t-test and verified with Pearson's chi-square test. A $p$ value of less than 0.05 was considered significant.

\section{Results}

\section{Socio-demographical profiles}

The average age of the partial laryngectomy group (Group 1) was 57.3 years, with a range between 42 and
67 years. In the total laryngectomy group (Group 2), the average age was 63.4 years, with range between 43 and 84 years. Statistical analysis showed no significant difference between the mean age of the groups $(p=0.093)$. The differences in the levels of education and average income between the groups was not significant $(p=0.084$ and $p=$ 0.088 , respectively).

\section{Analysis of impairment}

The average score on the QLQ-H\&N35 was higher in Group 2 than in Group 1 in all sections except weight increase. The sections in which the differences between the average scores reached statistical significance were swallowing (HNSW; Group 1 = 9.4; Group $2=21.4 ; p=$ 0.009 ), senses (HNSW; Group 1 $=11.6$; Group $2=47.7 ; p$ $=0.000)$, speech (HNSP; Group $1=44.6$; Group $2=59.7$; $p=0.038$ ), social eating (HNSO; Group $1=10.5$; Group $2=28.8 ; p=0.011$ ), sexuality (HNSX; Group $1=29.0$; Group $2=54.3 ; p=0.005$ ), problems with teeth (HNTE; Group $1=22.9$; Group $2=45.5 ; p=0.011$ ), problems with opening mouth (HNOM; Group $1=6.3$; Group $2=$ 28.6; $p=0.003$ ) and coughing (HNCO; Group $1=17.0$; Group $2=49.1 ; p=0.000$ ) (Table I).

The mean ASEX score was $13.6 \pm 5.1$ in Group 1 and $4.7 \pm 4.2$ in Group 2. The difference was not significant (ASEX1: $p=0.386$ ). In Group 1, 11 patients of 31 (35.4\%) indicated sexual dysfunction, with scores higher than 11. In Group 2, the total was 30 patients of $51(52.9 \%)$. The difference between the groups was remarkable, however, it was not statistically significant (ASEX2: $p=0.127$ ). (Table II).

Investigating the correlation between the results of ASEX and HNSX of Group 1 using the Pearson method, it can be observed that HNSX values were positively correlated with both ASEX1 and ASEX2 scores, which indicate sexual dysfunction $(r=0.771$ and $p=0.000 ; r=0.891$ and $p=0.000$, respectively). The same calculations for Group 2 indicate a high positive correlation between HNSX and $\operatorname{ASEX} 2(r=0.525$ and $p=0.000)$, but no correlation between HNSX and ASEX1 $(r=0.183$ and $p=0.199)$ (Tables II and IV).

\section{Discussion}

The most important outcome regarding the socio-demographic profiles of the patients was the absence of a significant difference between total laryngectomy and partial laryngectomy patients in terms of the mean age of the patients, occupation and average education and income. The homogeneity of the demographic characteristics prevented sociocultural factors from influencing outcomes. The average age was similar to the previous study by Tas et al ${ }^{5}$. The all-male sample set was inconsistent with the literature ${ }^{56}$, but another study that included only men is also available ${ }^{2}$. 
Table I. Mean Scores of the EORTC QLQ-H\&N35 items and scales of two groups and comparison.

\begin{tabular}{|c|c|c|c|}
\hline Symptom scales & $\begin{array}{c}\text { Group } 1 \\
\text { Partial laryngectomy }\end{array}$ & $\begin{array}{c}\text { Group } 2 \\
\text { Total laryngectomy }\end{array}$ & $P$ value \\
\hline HNPA (Pain) & 15.5 & 18.5 & 0.449 \\
\hline HNSW (Swallowing) & 9.4 & 21.4 & 0.009 \\
\hline HNSE (Senses) & 11.6 & 47.7 & 0.000 \\
\hline HNSP (Speech) & 44.6 & 59.7 & 0.038 \\
\hline HNSO (Social eating) & 10.5 & 28.8 & 0.011 \\
\hline HNSC (Social contact) & 19.1 & 32.3 & 0.055 \\
\hline HNSX (Sexuality) & 29.0 & 54.3 & 0.005 \\
\hline \multicolumn{4}{|l|}{ Symptom Items } \\
\hline HNTE (Problems with teeth) & 22.9 & 45.5 & 0.011 \\
\hline HNOM (Problems with opening mouth) & 6.3 & 28.6 & 0.003 \\
\hline HNDR (Dry mouth) & 26.0 & 31.8 & 0.425 \\
\hline HNSS (Sticky maliva) & 33.1 & 39.8 & 0.386 \\
\hline HNCO (Coughing) & 17.0 & 49.1 & 0.000 \\
\hline HNFI (Feeling ill) & 27.1 & 28.7 & 0.817 \\
\hline \multicolumn{4}{|l|}{ Dichotomous Items } \\
\hline HNPK (Use of painkillers) & 41.9 & 54.9 & 0.260 \\
\hline HNNU (Use of nutritional supplements) & 11.3 & 12.3 & 0.542 \\
\hline HNFE (Use of feeding tube) & 12.9 & 27.4 & 0.126 \\
\hline HNWL (Weight decrease) & 19.3 & 33.3 & 0.176 \\
\hline HNWG (Weight increase) & 54.8 & 47.0 & 0.135 \\
\hline
\end{tabular}

In several studies ${ }^{578}$, the speech parameter (HNSP) of the QLQ-H\&N35 questionnaire was considered the most important for indicating quality of life. Other studies validated the QLQH\&N35 for reflecting speech problems ${ }^{29}$. In the current study, we found significantly lower scores for speech problems in the partial laryngectomy group $(p=0.038)$. The data we obtained is mostly consistent with the literature, although we disagree with the assertion by Müller et al. ${ }^{8}$ that the QLQH\&N35 does not accurately reflect speech difficulties.

When other parameters of the QLQ-H\&N35 were considered, it was observed that the patients with total laryngectomy had more problems in swallowing (HNSW) than patients with partial laryngectomy, in addition to teeth (HNTE), opening their mouth (HNOM), sensation (HNSE), coughing (HNCO), social eating (HNSO) and sex (HNSX). Predictably, partial laryngectomy patients scored higher only in the weight increase (HNWG) section. Although they scored lower compared to total laryngectomy patients for pain, dry mouth, sticky saliva, feeling ill, use of painkillers, and trouble with social contact, the differences were not significant. We believe the data we obtained are plausible and self-confirming.

According to the study conducted by Braz et al. ${ }^{10}$, problems such as fatigue, smell and taste disorders, and avoidance of eating in social environments, occured more frequently in the total laryngectomy group than in the partial laryngectomy group; however, the difference was significant only for sensory problems. Similarly, Swnaik et
Table II. Mean scores of the Arizona sexual experiences scale of two groups and comparison.

\begin{tabular}{lccc} 
& $\begin{array}{c}\text { Group 1 } \\
\text { (Partial } \\
\text { laryngectomy; } \\
\mathbf{n = 3 1 )}\end{array}$ & $\begin{array}{c}\text { Group 2 } \\
\text { (Total } \\
\text { laryngectomy; } \\
\mathbf{n = 5 1 )}\end{array}$ & P value \\
ASEX 1 & 13.6 & 14.7 & 0.386 \\
ASEX 2 & 0.35 & 0.52 & 0.127 \\
\hline
\end{tabular}

Table III. Correlation between ASEX and HNSX scores of group $1(n=31)$.

$\begin{array}{llccc} & & \text { ASEX 1 } & \text { ASEX 2 } & \text { HNSX } \\ \text { ASEX } 1 & \text { Pearson correlation } & 1.000 & 0.713^{* *} & 0.771^{* *} \\ & p & & 0.000 & 0.000 \\ \text { ASEX } 2 & \text { Pearson correlation } & 0.713^{* *} & 1.000 & 0.891^{* *} \\ & p & 0.000 & & 0.000 \\ \text { HNSX } & \text { Pearson correlation } & 0.771^{* *} & 0.891^{* *} & 1.000 \\ & p & 0.000 & 0.000 & \end{array}$

${ }^{* *}$ Correlation is significant at the 0.01 level (2-tailed)

* Correlation is significant at the 0.05 level (2-tailed)

al. ${ }^{11}$, in their comparison of the quality of life after total and partial laryngectomy, found that only smell and taste disorders were higher in patients with total laryngectomy. Akduman et al. ${ }^{9}$ reported that, among all parameters of the QLQ-HN35, only speech problems and coughing scores were higher in total laryngectomy patients compared to partial laryngectomy patients. Furthermore, Fil- 
Table IV. Correlation between ASEX and HNSX scores of group $2(n=51)$.

\begin{tabular}{|c|c|c|c|c|}
\hline & & HNSX & ASEX 1 & ASEX 2 \\
\hline \multirow[t]{2}{*}{ HNSX } & Pearson correlation & 1.000 & 0.183 & 0.525 ** \\
\hline & $p$ & & 0.199 & 0.000 \\
\hline \multirow[t]{2}{*}{ ASEX 1} & Pearson correlation & 0.183 & 1.000 & $0.483^{* *}$ \\
\hline & $p$ & 0.199 & & 0.000 \\
\hline \multirow[t]{2}{*}{ ASEX 2} & Pearson correlation & 0.525 ** & 0.483 ** & 1.000 \\
\hline & $p$ & 0.000 & 0.000 & \\
\hline
\end{tabular}

${ }^{* *}$ Correlation is significant at the 0.01 level (2-tailed).

${ }^{*}$ Correlation is significant at the 0.05 level (2-tailed)

ipovski et al. ${ }^{2}$ found that speech, swallowing, sensation and coughing problems, as well as difficulty in establishing social relationships were significantly higher among patients with total laryngectomy. Our study reports significant differences between the two groups in the opening mouth, taste, and sex parameters, although other studies found no difference 2910 .

Sexuality following laryngectomy has not been evaluated in depth in previous studies ${ }^{12}$. Considering early investigations on sexual problems, Gardner's 1960 study ${ }^{13}$ on laryngectomies is one of the first. In that study, $23 \%$ of female patients reported that they felt less feminine and $35 \%$ that they felt less attractive post-surgery. In 1980, Myers et al. ${ }^{14}$ surveyed 48 laryngectomised patients of whom $82 \%$ had been sexually active before the operation. Approximately one in five of these patients felt less attractive, and one-third complained that their relationship was altered.

De Boer ${ }^{15}$ divided patients into three groups according to treatment, namely radiation, laryngectomy, or laryngectomy plus neck dissection. The laryngectomy patients reported a $44 \%$ reduction in sexual contact. Similarly, Harran and Gavilan ${ }^{16}$ studied 111 larynx cancer patients and found that partial laryngectomy patients had sexual problems more often than total laryngectomy patients. The de Boer study also found that women had more problems than men did. In contrast, Singer et al. ${ }^{12}$ used the QLQ-H\&N35 form to determine the differences between total and partial laryngectomy patients in the frequency of sexual difficulty, and the HNSX score for total laryngectomy patients was significantly higher than for partial laryngectomy patients.

Other recent studies about the psychology of laryngectomised patients focused on the quality of life for the spouses of patients. Offerman et al. ${ }^{17}$ and Meyer et al. ${ }^{18}$ reported that the spouses of patients who underwent total laryngectomy needed professional psychological support and that the surgery had an effect on their sexual relationships. Although our sample set did not include spouses, we think that our results are in accordance with these studies. We plan to study the spouses of total laryngec- tomised patients using the same questionnaires as in the current study.

The differences between the two groups in ASEX scores and in the number of patients with sexual dysfunction scores over 11 were not significant. However, we believe it is valuable to know that the average ASEX scores and the number of patients with sexual dysfunction in Group 2 were higher than in Group 1. Yilmaz et al. ${ }^{19}$ found results that were similar to ours.

In contrast to studies that found no significant difference between total and partial laryngectomy groups in terms of HNSX ${ }^{210}$, our data set revealed a highly significant difference in HNSX scores $(p<0.005)$ between the total and partial laryngectomy groups. This differed from other studies that showed significant differences between total and partial laryngectomy groups in terms of HNSX ${ }^{12}$. In our study, while the HNSX scores were significantly different between groups, the ASEX scores showed no sexual function difference. While this is an apparent contradiction, it can be explained by the fact that the QLQH\&N35 scale for HNSX primarily reflects libido and sexual enjoyment, and not sexual dysfunction ${ }^{12}$. The ASEX scale shows sexual dysfunction with tested reliability ${ }^{20}$.

We also evaluated the relationship of the sex scales with each other using Pearson's correlation and determined a highly significant correlation between the HNSX scale and the ASEX 2 scale $(p=0.000)$ in both the partial laryngectomy and total laryngectomy groups. Between the HNSX scale and ASEX1 scale there was a high correlation in the partial laryngectomy group $(p=0.000)$, but there was no significant correlation in the total laryngectomy group $(p=0.199)$. In both groups, there was a high correlation between ASEX1 and ASEX2 ( $p=$ 0.000).

These results deserve a special explanation. Primarily, although there was no significant difference in terms of the ASEX scale and there was a significant difference in terms of HNSX, the high correlation of both tests in both groups might be because the HNSX scale does not reflect sexual dysfunction fully, although it includes some elements of 
sexual function (libido and sexual enjoyment). Secondly, the detection of the total score from ASEX1 highly correlated with the presence of sexual dysfunction regarding ASEX2 in both groups suggests that ASEX1 could be used as a parameter for sexual dysfunction. Thirdly, the reason for the high correlation of the ASEX1 and HNSX scales in the partial laryngectomy group, while showing no correlation in the total laryngectomy group, might be because of the lack of verbal communication or because the HNSX scale and ASEX1 do not fully reflect sexual dysfunction. Lastly, in light of all these, HNSX may be a more appropriate method to compare these patient groups.

Our sample set was not large. This point can be seen as a weakness, but we think that our results are reliable and compatible with the recent literature. The epidemiological distribution of the study groups was similar and reduced the risk of bias.

\section{Conclusions}

These results show that the quality of life for patients who undergo total laryngectomy is lower than for those who undergo partial laryngectomy. Survival is, of course, the most important factor in the follow-up of patients with laryngeal cancer, but the psychosocial sequela of the disease and its treatment should not be underestimated.

The relationship between laryngectomy, sexuality and quality of life is a complex, intertwined issue. Many points remain to be clarified in future studies.

\section{References}

1 Bjordal F, Hammerlid M, Ahlner-Elmqvist M, et al. Quality of life in head and neck cancer patients: validation of the European Organization for Research and Treatment of Cancer Quality of Life Questionnaire - H\&N35. J Clin Oncol 1999;17:1008-19.

2 Filipovski A, Mušanović M, Hodžić D, et al. Quality of life in patients with larygeal / hypopharyngeal cancer following total/partial laryngectomy. Med Glas (Zenica) 2012; 9:287-92.

3 Bjordal K, Ahlner-Elmqvist M, Tolleson, et al. Development of a European Organization for Research and Treatment of Cancer (EORTC) questionnaire module to be used in head and neck cancer. Acta Oncol 1994;33:879-85.

4 Fayers PM, Aaronson NK, Bjordal K, et al. EORTC scoring manually. $3^{\text {rd }}$ ed. Brusssels: EORTC Quality of Life Group; 2001. p. 86.

5 Taş A, Yağiz R, Karasalihoğlu AR, et al. Assessment of quality of life in patients with laryngeal cancer after surgical treatment. Kulak Burun Bogaz Ihtis Derg 2004;12:84-90.
6 Nordgren M, Abendstein H, Jännert M, et al. Health-related quality of life five years after diagnosis of laryngeal carcinoma. Int J Radiat Oncol Biol Phys 2003;56:1333-43.

7 DeSanto LW, Olsen KD, Perry WC, et al. Quality of life after surgical treatment of cancer of the larynx. Ann Otol Rhinol Laryngol 1995;104:763-9.

8 Müller R, Paneff J, Köllner V, et al. Quality of life of patients with laryngeal carcinoma: a post-treatment study. Eur Arch Otorhinolaryngol 2001;258:276-80.

9 Akduman D, Karaman M, Uslu C, et al. Larynnx cancer treatment results: survive and quality of life assessment. $\mathrm{Ku}-$ lak Burun Bogaz Ihtis Derg 2010;20:25-32.

10 Braz DS, Ribas MM, Dedivitis RA, et al. Quality of life and depression in patients undergoing total and partial laryngectomy. Clinics (Sao Paulo) 2005;60:135-42.

11 Sewnaik A, van den Brink JL, Wieringa MH, et al. Surgery for recurrent laryngeal carcinoma after radiotherapy: partial laryngectomy or total laryngectomy for a better quality of life. Otolaryngol Head Neck Surg 2005;132:95-8.

12 Singer S, Danker H, Dietz A, et al. Sexual problems after total or partial laryngectomy. Laryngoscope 2008;118:2218-24.

13 Gardner WH. Adjustment problems of laryngectomy that woman. Arch Otolaryngol 1966;83:31-42.

14 Meyers AD, Aarons B, Suzuki W, et al. Following laryngectomy sexual behavior. Ear Nose Throat J 1980;59:327-9.

15 de Boer MF, Pruyn JF, van den Borne B, et al. Rehabilitation outcomes of long-term survivors treated for head and neck cancer. Head Neck 1995;17:503-15.

16 Herranz J, Gavilan J. Psychosocial adjustment after laryngeal cancer surgery. Ann Otol Rhinol Laryngol 1999;108: 990-7.

17 Offerman MP, Pruyn JF, de Boer MF, et al. Psychosocial consequences for partners of patients after total laryngectomy and for the relationship between patients and partners. Oral Oncol 2015;51:389-98.

18 Meyer A, Keszte J, Wollbrück D, et al. Psychological distress and need for psycho-oncological support in spouses of total laryngectomised cancer patients results for the first 3 years after surgery. Support Care Cancer 2015;23:1331-9.

19 Yilmaz M, Yener M, Yollu U, et al. Depression, self-esteem and sexual function in laryngeal cancer patients. Clin Otolaryngol 2015;40:349-54.

${ }^{20}$ McGahuey CA, Gelenberg AJ, Laukes CA, et al. The Arizona Sexual Experience Scale (ASEX): reliability and validity. J Sex Marital Ther 2000; 26:25-40. 